TRANSACTIONS OF THE

AMERICAN MATHEMATICAL SOCIETY

Volume 187, Issue 1, 1974

\title{
SOME POSITIVE TRIGONOMETRIC SUMS
}

\author{
BY
}

\section{RICHARD ASKEY( $\left.{ }^{1}\right)$ AND JOHN STEINIG}

\begin{abstract}
Vietoris found an interesting generalization of the classical inequality $\sum_{k=1}^{n}(\sin k \theta / k)>0,0<\theta<\pi$. A simplified proof is given for his inequality and his similar inequality for cosine series. Various new results which follow from these inequalities include improved estimates for the location of the zeros of a class of trigonometric polynomials and new positive sums of ultraspherical polynomials which extend an old inequality of Fejér. Both of Vietoris' inequalities are special cases of a general problem for Jacobi polynomials, and a summary is given of known results on this problem.
\end{abstract}

1. Vietoris' theorems. In a little known paper [18] Vietoris proved the following theorems.

Theorem 1. If $a_{0} \geq a_{1} \geq \cdots \geq a_{n}>0$ and $(2 k) a_{2 k} \leq(2 k-1) a_{2 k-1}, k \geq 1$, then

$$
s_{n}(x)=\sum_{k=1}^{n} a_{k} \sin k x>0, \quad 0<x<\pi,
$$

and

$$
t_{n}(x)=\sum_{k=0}^{n} a_{k} \cos k x>0, \quad 0<x<\pi
$$

Theorem 2. If

$$
c_{2 k}=c_{2 k+1}=2^{-2 k}\left(\begin{array}{c}
2 k \\
k
\end{array}\right), \quad k \geq 0,
$$

then

$$
\sigma_{n}(x)=\sum_{k=1}^{n} c_{k} \sin k x>0, \quad 0<x<\pi
$$

and

$$
\tau_{n}(x)=\sum_{k=0}^{n} c_{k} \cos k x>0, \quad 0<x<\pi .
$$

These two theorems are equivalent. The second is obviously a special case of the first. On the other hand, Theorem 1 follows from Theorem 2. For if

Received by the editors October 24, 1972 and, in revised form, February 6, 1973. AMS (MOS) subject classifications (1970). Primary 42A04; Secondary 26A78, 33A65. (1) Sponsored by the United States Army under Contract No. DA-31-124-ARO-D-462. 
$d_{0} \geq d_{1} \geq \cdots \geq d_{n}>0$, a summation by parts shows that

$$
\sum_{k=1}^{n} c_{k} d_{k} \sin k x>0, \quad 0<x<\pi,
$$

and

$$
\sum_{k=0}^{n} c_{k} d_{k} \cos k x>0, \quad 0<x<\pi .
$$

Letting $a_{k}=c_{k} d_{k}, 0 \leq k \leq n$, gives Theorem 1 .

For completeness we will give a proof of Theorem 2. Some of Vietoris' ideas will be used, but many of the difficulties of his proof have been replaced by easier arguments. However the reader should be aware that greater elegance often means diminished power, and Vietoris' original arguments may turn out to be useful for the more complicated problems which are mentioned at the end of this paper. The applications include estimates for the zeros of certain trigonometric polynomials and an improvement of a forty-year old result of Fejér on sums of ultraspherical polynomials.

For the proof we need three lemmas.

Lemma 1. For $m \geq 1$ we bave $(\underset{m}{2 m})<2^{2 m}(\pi m)^{-1 / 2}$.

Proof. Let $\gamma_{m}=m^{1 / 2} 2^{-2 m(2 m} \underset{m}{2 m}$. Then $\gamma_{m}<\gamma_{m+1}$ for $m \geq 1$; and by Stirling's formula, $\gamma_{m} \rightarrow \pi^{-1 / 2}$ as $m \rightarrow \infty$.

Lemma 2. If the sequence $\left\{c_{k}\right\}_{k=0}^{\infty}$ is defined by (1.1), then for $0<x<\pi$,

$$
\sum_{k=1}^{\infty} c_{k} \sin k x=\sum_{k=0}^{\infty} c_{k} \cos k x=\left(\frac{1}{2} \cot \frac{x}{2}\right)^{1 / 2} \text {. }
$$

Proof. For $|z| \leq 1, z \neq 1$, we have $(1-z)^{-1 / 2}=\Sigma_{k=0}^{\infty} c_{2 k} z^{k}$. Since $c_{2 k}=$ $c_{2 k+1}$, it follows that

$$
(1+z)\left(1-z^{2}\right)^{-1 / 2}=\sum_{k=0}^{\infty} c_{k} z^{k}
$$

for $|z| \leq 1, z \neq \pm 1$. On setting $z=e^{i x}, 0<x<\pi$, and separating real and imaginary parts, we get (1.4).

Lemma 3. Let $P_{r}(x)^{\circ}=\Sigma_{k=0}^{r} b_{k} e^{i k x}$, where $b_{0} \geq b_{1} \geq \cdots \geq b_{r}>0$. Then for $n>m \geq 0$ we have

$$
\left|P_{n}(x)-P_{m}(x)\right| \leq b_{m+1} / \sin (x / 2), \quad 0<N<2 \pi
$$

Proof. Sum by parts and use the standard estimate $\left|\Sigma_{k=0}^{n} e^{i k x}\right| \leq 1 / \sin (x / 2)$. We are now ready to prove Theorem 2 . 
Proof. Consider $\sigma_{n}$ first. We may assume $n \geq 2$. Different arguments are needed for each of the intervals $0<x \leq \pi / n, \pi / n<x<\pi-\pi / n$ and $\pi-\pi / n \leq x<\pi$.

For $0<x \leq \pi / n$, all terms in the sum are nonnegative, and the first is strictly positive.

For $\pi-\pi / n \leq x<\pi$, set $x=\pi-y$, so that $0<y \leq \pi / n$. If $n$ is even, say $n=2 m$, we have

$$
\begin{aligned}
\sigma_{n}(x) & =\sum_{k=1}^{2 m}(-1)^{k-1} c_{k} \sin k y=\sum_{k=1}^{m}\left[c_{2 k-1} \sin (2 k-1) y-c_{2 k} \sin 2 k y\right] \\
& =\sum_{k=1}^{m}(2 k-1) c_{2 k-1}\left[\frac{\sin (2 k-1) y}{2 k-1}-\frac{\sin 2 k y}{2 k}\right] .
\end{aligned}
$$

This last sum has positive terms, since $t^{-1} \sin t$ is decreasing in $t$ on $(0, \pi]$ and $2 k y \leq 2 m y=n y \leq \pi$. And if $n$ is odd there is an extra term, $c_{n} \sin n y$, which is positive for $0<y<\pi / n$.

If $n \geq 3$, we must still consider the interval $\pi / n<x<\pi-\pi / n$. There we have $\sin x>\sin (\pi / n) \geq(\pi / n)\left(1-\pi^{2} / 6 n^{2}\right)$. Now by Lemmas 2 and 3 ,

$$
\sigma_{n}(x) \geq(1 / 2 \cot (x / 2))^{1 / 2}-c_{n+1} / \sin (x / 2) ;
$$

hence, for $\pi / n<x<\pi-\pi / n$, we have

$$
2 \sin (x / 2) \sigma_{n}(x) \geq\left[(\pi / n)\left(1-\pi^{2} / 6 n^{2}\right)\right]^{1 / 2}-2 c_{n+1} .
$$

Here, the term in square brackets is decreasing in $n$ for $n \geq 3$, and $c_{2 m}=c_{2 m+1}$ for $m \geq 0$. Hence, the right hand side of (1.6) is positive for $n=2 m-1$, if it is positive for $n=2 m$. And for $n=2 m$ it follows from Lemma 1 that the right hand side of $(1.6)$ is at least equal to

$$
(2 \pi m)^{-1 / 2}\left\{\pi\left(1-\pi^{2} / 24 m^{2}\right)^{1 / 2}-2 \sqrt{2}\right\}
$$

this is positive for $m \geq 2$. Therefore $\sigma_{n}(x)>0$ for $\pi / n<x<\pi-\pi / n$. The theorem is now proven for $\sigma_{n}$.

For ${ }{ }_{n}$ the proof is similar to that for $\sigma_{n}$, but the details are slightly more involved. The result is obvious for $n=0$ and $n=1$, and an elementary computation shows that $r_{2}(x)=\cos ^{2} x+\cos x+1 / 2>0$. We may therefore assume $n \geq 3$.

Firstly, we observe that $\tau_{n}(x)>0$ for $0<x \leq \pi / n$, since

$$
\frac{d \tau_{n}}{d x}=-\sum_{k=1}^{n} k c_{k} \sin k x<0, \quad 0<x<\pi / n,
$$

and

$$
\tau_{n}\left(\frac{\pi}{n}\right)=\sum_{k=0}^{[n / 2]}\left(c_{k}-c_{n-k}\right) \cos \frac{k \pi}{n}>0
$$


Secondly, we show that ${ }_{n}(x)>0$ for $\pi-\pi /(n+1)<x<\pi$. We set $y=\pi-x$, and write

$$
\tau_{n}(x)=\sum_{k=0}^{[(n-1) / 2]} c_{2 k}[\cos 2 k y-\cos (2 k+1) y]+\epsilon_{n},
$$

where $\epsilon_{n}=0$ if $n=2 m-1$ and $\epsilon_{n}=c_{2 m} \cos 2 m y$ if $n=2 m$. When $\epsilon_{n}=0$, the monotonicity of $\cos x, 0 \leq x \leq \pi$, shows that $r_{n}(x)>0$ for $0<y<\pi / n$. When $n=2 m$, we have

$$
\begin{aligned}
\tau_{n}(x) & \geq c_{2 m}(1-\cos y+\cos 2 y-\cos 3 y+\cdots+\cos 2 m y) \\
& =c_{2 m}(1+\cos x+\cos 2 x+\cdots+\cos 2 m x) .
\end{aligned}
$$

Summing this series gives

$$
\tau_{n}(x) \geq c_{2 m} \frac{\sin (m+1 / 2) x \cos m x}{\sin (x / 2)}=c_{2 m} \frac{\cos (m+1 / 2) y \cos m y}{\cos (y / 2)} .
$$

It follows that $\tau_{n}(x)>0$ for $0<(m+1 / 2) y<\pi / 2$, that is, for $0<y<\pi /(n+1)$.

Lastly, we consider the interval $\pi /(n+1) \leq x \leq \pi-\pi /(n+1)$ for $n \geq 3$. The same argument as for $\sigma_{n}(x)$ on $\pi / n<x<\pi-\pi / n$ shows that it is enough if

$$
\left[\pi /(n+1)\left(1-\pi^{2} / 6(n+1)^{2}\right)\right]^{1 / 2}-2 c_{n+1}>0 .
$$

Here again, it suffices to consider even values of $n$, say $n=2 m$. Computation shows that this inequality holds for $n=4$ and 6 . For $m \geq 4$, the stronger inequality

$$
\left[\frac{\pi}{2 m+1}\left(1-\frac{\pi^{2}}{6(2 m+1)^{2}}\right)\right]^{1 / 2}-\frac{2}{\sqrt{\pi m}}>0
$$

holds, since it holds for $m=4$ and since its left hand side, when multiplied by $\sqrt{m}$, is an increasing function of $m$.

This concludes the proof for ${ }^{r} n$.

2. Applications to other trigonometric sums. A trigonometric polynomial with real coefficients, of degree $n$, has exactly $2 n$ zeros (modulo $2 \pi$ ) $[12, \S$ VI, Proplem 14]. Pólya [11] proved that a sine polynomial, or a cosine polynomial, with positive and monotonically increasing coefficients, has all its zeros real and simple. Then Szegö [15] showed how one could obtain estimates for the location of the zeros of such polynomials; this is the problem which we now consider.

We require the following corollary of Theorem 1.

Corollary 1. If $(2 k-1) A_{k-1} \geq 2 k A_{k}>0$ for $k \geq 1$, and if $0<x<2 \pi$, then

$$
\sum_{k=0}^{n} A_{k} \sin (k+1 / 4) x>0 \text { and } \sum_{k=0}^{n} A_{k} \cos (k+1 / 4) x>0 \text {. }
$$


Proof. Set $a_{k}=2^{-2 k}\left(\begin{array}{c}2 k \\ k\end{array}\right)$. It suffices to establish the particular case $A_{k}=a_{k}$, $k=0, \cdots, n$; the general case then follows by partial summation.

Now by Theorem 2, we have

$$
\begin{array}{ll}
\sum_{k=0}^{n} a_{k}[\sin 2 k x+\sin (2 k+1) x]>0, & 0<x<\pi, \\
\sum_{k=0}^{n} a_{k}[\cos 2 k x+\cos (2 k+1) x]>0, & 0<x<\pi .
\end{array}
$$

Hence,

$$
\sum_{k=0}^{n} a_{k} \sin (2 k+1 / 2) x>0 \text { and } \sum_{k=0}^{n} a_{k} \cos (2 k+1 / 2) x>0
$$

for $0<x<\pi$, and the proof is complete.

Corollary 2. Let $A_{1}, \cdots, A_{n}$ satisfy the conditions of Corollary 1. If $0 \leq \nu$ $\leq 1 / 4$ and $0<x<2 \pi$, or $-1 / 4 \leq \nu \leq 1 / 4$ and $0<x<\pi$, then $\sum_{k=0}^{n} A_{k} \cos (k+\nu) x>0$.

Proof. Use Corollary 1 , and the identity

$$
\cos (k+\nu) x=\cos (k+1 / 4) x \cos (\nu-1 / 4) x-\sin (k+1 / 4) x \sin (\nu-1 / 4) x .
$$

Similarly, one proves

Corollary 3. Let $A_{1}, \cdots, A_{n}$ satisfy the conditions of Corollary 1. If $1 / 4 \leq \nu$ $\leq 1 / 2$ and $0<x<2 \pi$, or $1 / 4 \leq \nu \leq 3 / 4$ and $0<x<\pi$, then $\sum_{k=0}^{n} A_{k} \sin (k+\nu) x \geq 0$.

We shall combine Corollary 1 with an argument due to Szegö, and obtain bounds for the zeros of a wide class of trigonometric polynomials. Szegö [15] applied the classical inequality of Fejér

$$
\sum_{k=0}^{n} \sin (k+1 / 2) x \geq 0, \quad 0 \leq x \leq 2 \pi
$$

to prove, among other things, the following two theorems.

Theorem A. If $\lambda_{0}>\lambda_{1} \geq \lambda_{2} \geq \cdots \geq \lambda_{n} \geq 0$, and if $s_{k}, t_{k}$ denote the zeros of

and of

$$
p(t)=\lambda_{0} \cos n t+\lambda_{1} \cos (n-1) t+\cdots+\lambda_{n-1} \cos t+\lambda_{n}
$$

$$
q(t)=\lambda_{0} \sin n t+\lambda_{1} \sin (n-1) t+\cdots+\lambda_{n-1} \sin t,
$$

respectively, on $0<t<\pi$, then

$$
\begin{aligned}
& (k-1 / 2) \pi /(n+1 / 2)<s_{k}<(k+1 / 2) \pi /(n+1 / 2), \quad k=1, \ldots, n, \\
& k \pi /(n+1 / 2)<t_{k}<(k+1) \pi /(n+1 / 2), \quad k=1, \ldots, n-1 .
\end{aligned}
$$


Theorem B. If, in addition to the conditions in Theorem A, we bave

$$
2 \lambda_{0}-\lambda_{1}>\lambda_{1}-\lambda_{2} \geq \lambda_{2}-\lambda_{3} \geq \cdots \geq \lambda_{n-1}-\lambda_{n} \geq \lambda_{n} \geq 0,
$$

then the right band sides of (2.2) and (2.3) can be replaced by $k \pi / n$ and $(k+1 / 2) \pi / n$, respectively.

It is interesting to remark at this juncture that one may not replace $\sin (k+1 / 2) x$ by $\cos (k+1 / 2) x$ in $(2.1)$, since

$$
\sum_{k=0}^{n} \cos (k+1 / 2) x=\frac{\sin (n+1) x}{2 \sin (x / 2)}, \quad 0<x<2 \pi .
$$

In contrast, we have at our disposal in Corollary 1 a sine polynomial and the corresponding cosine polynomial, both positive on $(0,2 \pi)$. This allows us to strengthen both sides of the inequalities in Theorem $A$ under a supplementary condition on the $\lambda$ 's different from the one in Theorem $B$. We shall prove

Theorem 3. Let $p(t), q(t), s_{k}$ and $t_{k}$ be defined as in Theorem A. If

$$
(2 k-1) \lambda_{k-1} \geq 2 k \lambda_{k}>0, \quad k \geq 1 \text {, }
$$

then

$$
\begin{gathered}
(k-1 / 2) \pi /(n+1 / 4)<s_{k}<k \pi /(n+1 / 4), \quad k=1, \ldots, n_{m} \\
k \pi /(n+1 / 4)<t_{k}<(k+1 / 2) \pi /(n+1 / 4), \quad k=1, \ldots, n-1 .
\end{gathered}
$$

Proof. Since $p(t)+i q(t)=\Sigma_{k=0}^{n} \lambda_{k} e^{i(n-k) t}$, we have

$$
e^{-i(n+1 / 4) t}[p(t)+i q(t)]=\sum_{k=0}^{n} \lambda_{k} \cos (k+1 / 4) t-i \sum_{k=0}^{n} \lambda_{k} \sin (k+1 / 4) t,
$$

whence

$$
\begin{aligned}
& p(t) \cos (n+1 / 4) t+q(t) \sin (n+1 / 4) t=\sum_{k=0}^{n} \lambda_{k} \cos (k+1 / 4) t, \\
& p(t) \sin (n+1 / 4) t-q(t) \cos (n+1 / 4) t=\sum_{k=0}^{n} \lambda_{k} \sin (k+1 / 4) t .
\end{aligned}
$$

By Corollary 1, therefore,

$$
\begin{array}{ll}
p(t) \cos (n+1 / 4) t+q(t) \sin (n+1 / 4) t>0, & 0<t<2 \pi, \\
p(t) \sin (n+1 / 4) t-q(t) \cos (n+1 / 4) t>0, & 0<t<2 \pi .
\end{array}
$$

Letting $t=k \pi /(n+1 / 4)$ in (2.8) and $t=(k+1 / 2) \pi /(n+1 / 4)$ in (2.9) leads to

$$
\begin{aligned}
(-1)^{k} p(k \pi /(n+1 / 4)) & >0, & k & =0,1, \ldots, n, \\
(-1)^{k} p((k+1 / 2) \pi /(n+1 / 4)) & >0, & k & =0,1, \ldots, n-1 ;
\end{aligned}
$$

and these inequalities imply (2.6). Similarly, we see that (2.7) holds, and the theorem is proved. 
The other zeros of $p(t)$ are at $t=2 m \pi \pm s_{k}$ and those of $q(t)$, at $t=2 m \pi \pm t_{k}$ and at $t=m \pi(m=0, \pm 1, \pm 2, \ldots)$.

The left hand inequalities in (2.6) and (2.7) are only slight improvements over the corresponding inequalities in Theorem $A$, but the right hand inequalities are slight improvements even on Theorem B. The condition (2.4) implies $\lambda_{k} \geq c(n-k)$ for some $c>0$, while the condition (2.5) in Theorem 3 implies only $\lambda_{k} \geq c^{\prime}(n-k)^{1 / 2}$. This gives some indication of the depth of Vietoris' theorem.

3. Applications to ultraspherical polynomials. Both the Fejér-Jackson-Gronwall inequality,

$$
\sum_{k=1}^{n} \frac{\sin k x}{k}>0, \quad 0<x<\pi,
$$

and W. H. Young's inequality,

$$
1+\sum_{k=1}^{n} \frac{\cos k x}{k}>0, \quad 0<x<\pi
$$

are contained in Theorem 1.

Since (3.1) implies or is related to many other inequalities (see for example [2], [8] and the references given there), the existence of an extension of (3.1) suggests that some of these other inequalities can also be extended. This is true to some extent, as we proceed to show.

Certain power series occur so frequently that their coefficients have acquired names and have been studied in detail. One such series is

$$
\left(1-2 x r+r^{2}\right)^{-\nu}=\sum_{n=0}^{\infty} C_{n}^{\nu}(x) r^{n}
$$

where $C_{n}^{\nu}(x)$ is a polynomial of degree $n$ in $x$. These are the ultraspherical polynomials; many of their properties are discussed in Chapter 4 of [16] and Chapter 10 of [6].

Fejér [7] proved that, for $n=1,2, \ldots$,

$$
\sum_{k=0}^{n} C_{k}^{\lambda}(x)>0, \quad-1<x<1,0<\lambda \leq 1 / 2 ;
$$

in other words, the power series coefficients of $(1-r)^{-1}\left(1-2 x r+r^{2}\right)^{-\lambda}$ are positive if $0<\lambda \leq 1 / 2$. One extension of this result was proved in [4]:

Theorem 4. The function $(1-r)^{-2 \nu}\left(1-2 x r+r^{2}\right)^{-\nu}$ bas positive power series coefficients for $-1<x<1, \nu>0$.

This theorem is equivalent to

$$
\sum_{k=0}^{n} \frac{(2 \nu)_{n-k}}{\left(n^{0}-k\right) !} C_{k}^{\nu}(x)>0, \quad-1<x<1, \nu>0 .
$$


Here, $(a)_{k}$ is defined by $(a)_{k}=\Gamma(a+k) / \Gamma(a)=a(a+1) \ldots(a+k-1)$; observe that $a_{k}=2^{-2 k}\left(\begin{array}{c}2 k \\ k\end{array}\right)=(1 / 2)_{k} / k !$.

A different extension of Fejér's inequality (3.2) is

Theorem 5. For $0<\nu \leq 1 / 4, \sum_{k=0}^{n}(1 / 2)_{k} C_{k}^{\nu}(x) /(2 \nu)_{k}>0,-1<x<1$.

Proof. Combine Corollary 2 with the following Mehler-type integral representation for $C_{n}^{\nu}(x)[5,3.15 .2(23)]$ :

$(\sin \theta)^{2 \nu-1} C_{n}^{\nu}(\cos \theta)=\frac{2^{\nu} \Gamma(\nu+1 / 2)}{\Gamma(1 / 2) \Gamma(\nu)} \frac{(2 \nu)}{n !} \int_{0}^{\theta}[\cos \phi-\cos \theta]^{\nu-1} \cos (n+\nu) \phi d \phi$, $\nu>0,0<\theta<\pi$.

A summation by parts shows that Theorem 5 is stronger than (3.2) for $0<\nu<1 / 4$.

A consequence of the case $\lambda=1 / 2$ of (3.2) is

$$
\sum_{k=0}^{n} \frac{C_{k}^{\nu}(x)}{C_{k}^{\nu}(1)}>0, \quad-1<x<1, \nu \geq 1 / 2 .
$$

This follows from Feldheim's integral [8],

$$
\begin{array}{r}
\frac{C_{n}^{\nu}(\cos \theta)}{C_{n}^{\nu}(1)}=\frac{2 \Gamma(\nu+1 / 2)}{\Gamma(\lambda+1 / 2) \Gamma(\nu-\lambda)} \int_{0}^{\pi / 2} \sin ^{2 \lambda} \phi \cos ^{2 \nu-2 \lambda-1} \phi\left(1-\sin ^{2} \theta \cos ^{2} \phi\right)^{n / 2} \\
\cdot \frac{C_{n}^{\lambda}\left(\cos \theta\left(1-\sin ^{2} \theta \cos ^{2} \phi\right)^{-1 / 2}\right)}{C_{n}^{\lambda}(1)} d \phi, \\
\nu>\lambda>-1 / 2,0<\theta<\pi,
\end{array}
$$

which shows that if (3.3) holds for some value of $\nu, \nu>-1 / 2$, then it holds for all larger values of $\nu$. For $\nu \geq 1,(3.3)$ follows from Feldheim's integral and (3.1), since

$$
C_{n}^{1}(\cos \theta) / C_{n}^{1}(1)=(\sin (n+1) \theta) /(n+1) \sin \theta .
$$

Using Feldheim's integral, (1.2) and (3.4) give

$$
\sum_{k=0}^{n}(k+1) c_{k+1} \frac{C_{k}^{\nu}(x)}{C_{k}^{\nu}(1)}>0, \quad-1<x<1, \nu \geq 1,
$$

where $c_{2 k}=c_{2 k+1}=(1 / 2)_{k} / k$ !. Since $k c_{k}<(k+1) c_{k+1}$ for $k \geq 1$, (3.5) implies (3.3) for $v \geq 1$, by partial summation.

An inequality in the same vein as (3.3) is

Theorem 6. For $\nu>0$ and $-1<x<1$, we bave

$$
\sum_{k=0}^{n} c_{k} \frac{C_{k}^{\nu}(x)}{C_{k}^{\nu}(1)}>0
$$

where $c_{2 k}=c_{2 k+1}=(1 / 2)_{k} / k !$ 
The proof follows immediately from Feldheim's integral and Theorem 2, since $\lim _{\lambda \rightarrow 0}\left(C_{n}^{\lambda}(\cos \theta) / C_{n}^{\lambda}(1)\right)=\cos n \theta$. This result was pointed out to us by George Gasper. It is weaker than (3.3) for $\nu \geq 1 / 2$.

4. Open problems. To fit Vietoris' theorem into a general context it is necessary to introduce Jacobi polynomials, $P_{n}^{(a, \beta)}(x)$, which may be defined by

(4.1) $(1-x)^{a}(1+x)^{\beta} P_{n}^{(\alpha, \beta)}(x)=\frac{(-1)^{n}}{2^{n} n !} \frac{d^{n}}{d x^{n}}\left[(1-x)^{n+a}(1+x)^{n+\beta}\right], \quad a, \beta>-1$.

They satisfy the orthogonality relations

$$
\begin{aligned}
& \int_{-1}^{1} P_{n}^{(\alpha, \beta)}(x) P_{m}^{(\alpha, \beta)}(x)(1-x)^{a}(1+x)^{\beta} d x=0, \quad m \neq n, \\
& \int_{-1}^{1}\left[P_{n}^{(a, \beta)}(x)\right]^{2}(1-x)^{a}(1+x)^{\beta} d x
\end{aligned}
$$

$$
=\frac{2^{a+\beta+1}}{(2 n+\alpha+\beta+1)} \frac{\Gamma(n+\alpha+1) \Gamma(n+\beta+1)}{\Gamma(n+1) \Gamma(n+\alpha+\beta+1)}=\left[b_{n}^{(a, \beta)}\right]^{-1} .
$$

The standard references are [16, Chapter 4] and [6, Chapter 10].

Vietoris' theorem and many other results are special cases of the following problem.

Problem 1. Expand $f(x)=(1-x)^{-\gamma}(1-x)^{-\delta}$ in an orthogonal series of Jacobi polynomials,

$$
f(x) \sim \sum_{n=0}^{\infty} a_{n} b_{n}^{(a, \beta)} P_{n}^{(\alpha, \beta)}(x)
$$

where

$$
a_{n}=\int_{-1}^{1}(1-x)^{-\gamma}(1+x)^{-\delta} P_{n}^{(a, \beta)}(x)(1-x)^{a}(1+x)^{\beta} d x
$$

For which values of $\alpha, \beta, \gamma, \delta$ are all the partial sums of (4.4) nonnegative, i.e.

$$
\sum_{k=0}^{n} a_{k} b_{k}^{(a, \beta)} P_{k}^{(a, \beta)}(x) \geq 0, \quad-1 \leq x \leq 1, n=0,1, \ldots \text { ? }
$$

Since

$$
P_{n}^{(-1 / 2,-1 / 2)}(\cos \theta) / P_{n}^{(-1 / 2,-1 / 2)}(1)=\cos n \theta
$$

and

$$
P_{n}^{(1 / 2,1 / 2)}(\cos \theta) / P_{n}^{(1 / 2,1 / 2)}(1)=(\sin (n+1) \theta) /(n+i) \sin \theta,
$$

Lemma 2 and Theorem 2 give a positive solution to Problem 1 for

$$
\begin{aligned}
& \alpha=\beta=-1 / 2, \quad \gamma=1 / 4, \quad \delta=-1 / 4, \\
& a=\beta=1 / 2, \quad \gamma=3 / 4, \quad \delta=1 / 4 .
\end{aligned}
$$

The case $a=\gamma, \beta=\delta$ has been studied in some detail. Problem 1 has a positive solution for $a=\beta,-1<\alpha \leq 3 / 2$, [16, Theorem 15.5] and [3], a negative solution for $\alpha>3 / 2$; and the general case $(\alpha, \beta)$ has a positive solution for 
$\alpha, \beta \geq 0, \alpha+\beta \leq 1 ; \alpha=\beta+1,-1 / 2 \leq \beta \leq 0 ; \beta=\alpha+1,-1 / 2 \leq \alpha \leq 0$ [1]; and a negative solution for $\alpha>\beta+1$ or $\beta>\alpha+1$ [1], and also for $a>3 / 2$ or $\beta>3 / 2$ [16, Theorem 15.2.2 and Theorem 15.4].

Other known cases of Problem 1 are

$$
\alpha=1 / 2, \quad \beta=-1 / 2, \quad \gamma=1, \quad \delta=0,
$$

which reduces to Fejêr's sum

$$
\sum_{k=0}^{n} \sin (k+1 / 2) \theta \geq 0
$$

and

$$
\alpha=\beta, \quad-1<\alpha \leq 0, \quad \gamma=\alpha+1 / 2, \quad \delta=0,
$$

which was proven by Fejér [7] for $-1 / 2 \leq a \leq 0$ and Szegö [17] for $-1<a<-1 / 2$.

Most of the methods which have been used to obtain positive results for Problem 1 are special and do not extend to other cases. The one exception is the use of the positive sum [2],

$$
\sum_{k=0}^{n} \frac{P_{k}^{(\alpha, \beta)}(x)}{P_{k}^{(\beta, a)}(1)}>0, \quad-1<x \leq 1,-1<a \leq \beta+1, a+\beta \geq 0 .
$$

This inequality is probably true for $\beta \geq-1 / 2, a+\beta \geq 0$, and if so it would give a new powerful weapon to attack special cases of Problem 1. In particular (4.12) for $a=3 / 2, \beta=-1 / 2$ is equivalent to

$$
\sum_{k=0}^{n}(k+1)^{2} \frac{P_{2 k}^{(3 / 2,3 / 2)}(x)}{P_{2 k}^{(3 / 2,3 / 2)}(1)} \geq \frac{1}{4} \sum_{k=0}^{n} \frac{P_{2 k}^{(3 / 2,3 / 2)}(x)}{P_{2 k}^{(3 / 2,3 / 2)}(1)}, \quad-1 \leq x \leq 1,
$$

and Problem 1 for $\alpha=\beta=\gamma=\delta=3 / 2$ is

$$
\sum_{k=0}^{n}(k+1)^{2} \frac{P_{2 k}^{(3 / 2,3 / 2)}(x)}{P_{2 k}^{(3 / 2,3 / 2)}(1)} \geq 0, \quad-1 \leq x \leq 1 .
$$

Since

$$
\sum_{k=0}^{n} \frac{P_{2 k}^{(3 / 2,3 / 2)}(x)}{P_{2 k}^{(3 / 2,3 / 2)}(1)}>0, \quad-1 \leq x \leq 1,
$$

(this is the even part of (3.3) for $\nu=2$ ), it is clear that (4.13) is a deeper result than (4.14). However (4.12) is not true for all $(\alpha, \beta)$. For instance, it fails for $a<1 / 2, \beta=-1 / 2$, when $n=2$.

Rather than leave the impression that all of these problems would be solved if only (4.12) held, we, should remark that Problem 1 can contain deeper results than (4.12). For example, when $\alpha=\beta=1 / 2,(4.12)$ is just the Fejér-Jackson-Gronwall inequality, and Vietoris' inequality (1.2) is a deeper result. Inequality (4.12) 
is deeper the closer $\beta$ is to $-1 / 2$ but unfortunately it is also harder to prove then. The common source of these two problems seems to be the following problem about Bessel functions:

Problem 2. For which values of $(\alpha, \beta)$ is

$$
\int_{0}^{x} t^{-\beta} J_{\alpha}(t) d t \geq 0, \quad x \geq 0 \text { ? }
$$

The condition $\beta<\alpha+1$ is necessary for the convergence of (4.15) at zero, and will not always be mentioned below.

The connection between (4.12) and Problem 2 comes from

$$
\lim _{n \rightarrow \infty} \frac{P_{n}^{(\alpha, \beta)}(\cos (\theta / n))}{P_{n}^{\left(a_{0} \beta\right)}(1)}=2^{a} \Gamma(\alpha+1) \theta^{-\alpha} J_{a}(\theta)
$$

If (4.12) holds for a pair $(\alpha, \beta)$ then Problem 2 has a positive solution for the same $(\alpha, \beta)$. The connection between Problems 1 and 2 comes from

$$
x^{\beta-1}=\frac{\int_{0}^{\infty} t^{-\beta} J_{a}(x t) d t}{\int_{0}^{\infty} t^{-\beta} J_{a}(t) d t} .
$$

Problem 2 is much more tractable than either of the problems involving Jacobi polynomials. When $\alpha \geq 1 / 2$, (4.15) holds for $\beta=-1 / 2$ (and hence for $\beta \geq-1 / 2$, by the second mean value theorem) by a theorem of Makai ([10], [13]); and the left hand side of (4.15) changes sign infinitely often on $0<x<\infty$ if $\beta<-1 / 2$ [14]. For -1 $<\alpha<1 / 2$, there is a unique solution $\beta(\alpha)(-1 / 2<\beta(\alpha)<0)$ to the equation

$$
\int_{0}^{j_{a}, 2} t^{-\beta(a)} J_{a}(t) d t=0
$$

where $j_{a, 2}$ is the second positive zero of $J_{a}(t)$. Then (4.15) holds for $\beta(\alpha) \leq \beta<$ $\alpha+1$ and the only cases of equality are $x=0$ or $\beta=\beta(\alpha), x=j_{a, 2}$. This has been proved by $\mathrm{E}$. Makai when $-1 / 2<\alpha<1 / 2$, it is classical when $\alpha=-1 / 2$, and the proof for $-1<a<-1 / 2$ follows. Makai's proof will appear in the near future.

Let $j_{a, n}$ denote the $n$th positive zero of $J_{a}(x)$. Then Makai's comparison theorem [10] applied to the differential equation

$$
y^{\prime \prime}+\left[\left(4 x^{2}-4 a^{2}+1\right) / 4 x^{2}\right] y=0, \quad y=x^{1 / 2} J_{a}(x),
$$

implies

$$
\left|\int_{j_{a, n-1}}^{j} x^{1 / 2} J_{a}(x) d x\right|>\left|\int_{j_{a, n}}^{j} x^{1 / n+1} J_{a}(x) d x\right|, \quad n=2,3, \ldots,|a|>1 / 2 .
$$

The second mean value theorem then gives

$$
\begin{aligned}
&\left|\int_{j_{a, n-1}}^{j} x^{-\beta} J_{a}(x) d x\right|> \mid \int_{j_{a, n}}^{j} x^{-\beta}, n+1 \\
& J_{a}(x) d x \mid, \\
& n=2,3, \cdots,-1<\alpha<-1 / 2,-1 / 2<\beta<0 .
\end{aligned}
$$


The proof of (4.15) for $-1<a<-1 / 2$ will be complete if we show that $\beta(\alpha)$ defined by (4.17) exists, $-1 / 2<\beta(\alpha)<0$, and is unique. Let

$$
g(\beta)=\int_{0}^{j} a,{ }_{t}-\beta J_{a}(t) d t .
$$

By Cooke's theorem (see [13]), $g(0)>0$, and we will show that $g(-1-\alpha)<0$. This gives the existence of $\beta(\alpha)$ and the inequalities $-1 / 2<-1-\alpha<\beta(\alpha)<0,-1<\alpha<$ $-1 / 2$.

Recall the classical differentiation formula $[6,7.2 .8(50)]$

$$
\frac{d}{d t}\left\{t^{a+1} J_{a+1}(t)\right\}=t^{a+1} J_{a}(t) \text {. }
$$

Integrating this gives

$$
\int_{0}^{j}{ }^{a, 2} t^{a+1} J_{a}(t) d t=\left(j_{a, 2}\right)^{a+1} J_{a+1}\left(j_{a, 2}\right), \quad \alpha>-1 .
$$

Now $J_{a+1}\left(j_{a, 2}\right)<0$ since for $a>-1$ we have $J_{a+1}(x)<0, j_{a+1,1}<x<j_{a+1,2}$ and $j_{a+1,1}<j_{a, 2}<j_{a+1,2}$. Thus there is at least one $\beta(\alpha)$, and $\int_{0}^{x} t-\beta(a) J_{\alpha}(t) d t>0$, $x \neq j_{a, 2}$, follows from the above argument. Then an integration by parts shows that $\int_{0}^{x} t^{-\beta} J_{a}(t) d t>0, x>0, \beta>\beta(\alpha)$, so $\beta(\alpha)$ is unique.

It is likely that $\beta(\alpha)$ is a decreasing function of $\alpha$ for $-1<\alpha<-1 / 2$. The following values of $\beta(\alpha)$ were computed by $\mathrm{J}$. Al-Abdulla using an integration package supplied by $C$. de Boor.

$$
\begin{array}{cc}
\alpha & \beta(\alpha) \\
-.5 & -.1915562 \\
-.4 & -.2259427 \\
-.3 & -.2593436 \\
-.2 & -.2918541 \\
-.1 & -.3235531 \\
0 & -.3545096 \\
.1 & -.3847832 \\
.2 & -.4144258 \\
.3 & -.4434834 \\
.4 & -.4719960 \\
.5 & -.5000000
\end{array}
$$

Makai's result gives a new proof of a theorem of Szegö [8]. Szegö proved that

$$
\int_{0}^{x} t^{-a} J_{a}(t) d t \geq 0, \quad a \geq \bar{a}, x \geq 0,
$$


where $\bar{a}$ is the solution of

$$
\int_{0}^{j} a,{ }^{2}-{ }^{a} J_{a}(t) d t=0
$$

Let $R$ be the set of $(\alpha, \beta)$ for which (4.15) holds. When $-1<a \leq 1 / 2$ the boundary of $R$ is the set of points where (4.17) holds, and from Sonine's first integral $R$ contains $(\alpha+\mu, \beta+\mu), \mu>0$, when it contains $(\alpha, \beta)$. See the argument in [8]. Another proof of Szegö's theorem is given in [9].

\section{REFERENCES}

1. R. Askey, Positivity of the Cotes numbers for some Jacobi abscissas, Numer. Math. 19 (1972), 46-48.

2. - Positive Jacobi polynomial sums, Tôhoku J. Math. 24 (1972), 109-119.

3. R. Askey and J. Fitch, Positivity of the Cotes numbers for some ultraspherical abscissas, SIAM J. Numer. Anal. 5 (1968), 199-201. MR 37 \#3750.

4. R. Askey and H. Pollard, Some absolutely monotonic and completely monotonic functions, SIAM J. Math. Anal. 5 (1974).

5. A. Erdélyi et al., Higher transcendental functions. Vol. 1, McGraw-Hill, New York, 1953. MR 15, 419.

6. - Higher transcendental functions. Vol. 2, McGraw-Hill, New York, 1953. MR 15, 419.

7. L. Fejér, Ultrasphärikus polynomok összegeröl, Mat. Fiz. Lapok 38 (1931), 161164 = Über die Summe ultrasphärischer Polynome, Gesammelte Arbeiten II, 421-423.

8. E. Feldheim, On the positivity of certain sums of ultraspherical polynomials, J. Analyse Math. 11 (1963), 275-284. MR 28 \#1333.

9. L. Lorch, M. E. Muldoon and P. Szego, Some monotonicity properties of Bessel functions, SIAM J. Math. Anal. 4 (1973), 385-392.

10. E. Makai, On a monotonic property of certain Sturm-Liouville functions, Acta Math. Acad. Sci. Hungar. 3 (1952), 165-172. MR 14, 872.

11. G. Pólya, Über die Nullstellen gewisser ganzer Funktionen, Math. Z. 2 (1918), 352-383.

12. G. Pólya and G. Szegö, Aufgaben und Lehrsätze aus der Analysis, Band II, Springer, Berlin, 1925.

13. J. Steinig, On a monotonicity property of Bessel functions, Math. Z. 122 (1971), 363-365.

14. - The sign of Lommel's function, Trans. Amer. Math. Soc. 163 (1971), 123-129. MR 44 \#1849.

15. G. Szegö, Inequalities for the zeros of Legendre polynomials and related functions, Trans. Amer. Math. Soc. 39 (1936), 1-17.

16. - Orthogonal polynomials, Amer. Math. Soc. Colloq. Publ., vol. 23, Amer. Math. Soc., Providence, R. I., 1967.

17. - Ultrasphaerikus polinomok összegeröl, Mat. Fiz. Lapok 45 (1938), 36-38.

18. L. Vietoris, Über das Vorzeichen gewisser trigonometrischer Summen, S.-B. Öst. Akad. Wiss. 167 (1958), 125-135; Anzeiger Öst. Akad. Wiss. 1959, 192-193.

DEPARTMENT OF MATHEMATICS, UNIVERSITY OF WISCONSIN, MADISON, WISCONSIN 53706 DÉPARTEMENT DE MATHÉMATIQUES, UNIVERSITÉ DE GENEVE, GENEVE, SWITZERLAND 